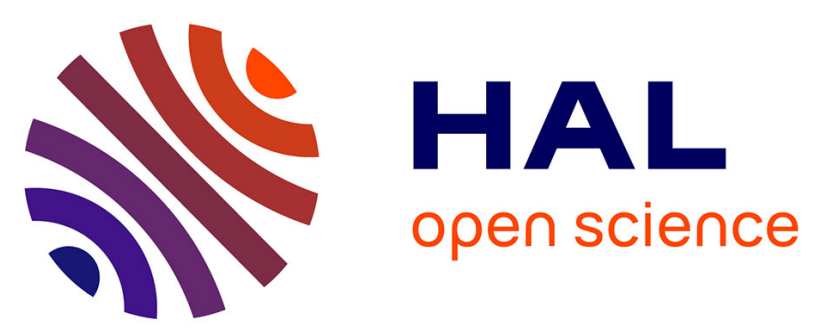

\title{
Topography, composition and structure of incipient Randall's plaque at the nanoscale level
}

Cécile Verrier, Dominique Bazin, Léa Huguet, Odile Stéphan, Alexandre

Gloter, Marie-Christine Verpont, Vincent Frochot, Jean-Philippe Haymann, Isabelle Brocheriou, Olivier Traxer, et al.

\section{To cite this version:}

Cécile Verrier, Dominique Bazin, Léa Huguet, Odile Stéphan, Alexandre Gloter, et al.. Topography, composition and structure of incipient Randall's plaque at the nanoscale level. Journal of Urology, 2016, 196 (5), pp.1566-1574. 10.1016/j.juro.2016.04.086 . hal-01313117

\section{HAL Id: hal-01313117 https://hal.sorbonne-universite.fr/hal-01313117}

Submitted on 9 May 2016

HAL is a multi-disciplinary open access archive for the deposit and dissemination of scientific research documents, whether they are published or not. The documents may come from teaching and research institutions in France or abroad, or from public or private research centers.
L'archive ouverte pluridisciplinaire HAL, est destinée au dépôt et à la diffusion de documents scientifiques de niveau recherche, publiés ou non, émanant des établissements d'enseignement et de recherche français ou étrangers, des laboratoires publics ou privés. 
Topography, composition and structure of incipient Randall's plaque at the nanoscale level

Cécile Verrier, ${ }^{1,2,3}$ Dominique Bazin, ${ }^{4,5}$ Léa Huguet, ${ }^{1,2}$ Odile Stéphan, ${ }^{5}$ Alexandre Gloter, ${ }^{5}$ Marie-Christine Verpont, ${ }^{1,2}$ Vincent Frochot, ${ }^{6}$ Jean-Philippe Haymann, ${ }^{1,2,6}$ Isabelle Brocheriou, ${ }^{1,2,7}$ Olivier Traxer, ${ }^{1,8}$ Michel Daudon, ${ }^{1,2,6}$ Emmanuel Letavernier, ${ }^{1,2,6}$

${ }^{1}$ Sorbonne Universités, UPMC Univ Paris 06, UMR S 1155, F-75020, Paris, France

${ }^{2}$ INSERM, UMR S 1155, F-75020, Paris, France

${ }^{3}$ Urology Unit, AP-HP, Hôpital Saint-Louis, F-75011, Paris, France

${ }^{4}$ CNRS, Laboratoire de Chimie de la Matière Condensée de Paris, UPMC, Collège de France, Paris, France

${ }^{5}$ Laboratoire de Physique des Solides, CNRS UMR 8502, Université Paris Sud XI, 91405 Orsay, France.

${ }^{6}$ Physiology Unit, AP-HP, Hôpital Tenon, F-75020, Paris, France

${ }^{7}$ Pathology Unit, AP-HP, Hôpital Tenon, F-75020, Paris, France

${ }^{8}$ Urology Unit, AP-HP, Hôpital Tenon, F-75020, Paris, France

\section{AUTHOR INFORMATION}

Correspondence:

Emmanuel LETAVERNIER, Service des Explorations Fonctionnelles Multidisciplinaires, Hôpital TENON, 4 rue de la Chine, 75020 Paris (France). Phone 33156016773 ; Fax 331 560170 03. E-mail: emmanuel.letavernier@tnn.aphp.fr

The authors declare no conflict of interest

Running Title: Incipient Randall's plaque

Word Count: 2993

Number of Figures and Tables: Figures:6; Table:1

Keywords: Randall's plaque, kidney stones, calcium, phosphate

Disclosure Statement: The authors have nothing to disclose 


\section{Abstract}

Purpose: Alexander Randall identified calcium phosphate plaques in renal papillae as the origin of kidney stones. However, little is known about the early steps of Randall's plaque formation, preceding the onset of urolithiasis. Our objectives were to characterize the composition and the initial formation site of incipient Randall's plaque in non-stone formers living patients.

Material and Methods: Median age of the patients was 67.7 years. Fifty-four healthy papillae from kidneys removed for cancer and without stones have been analyzed by immunohistochemistry and Von Kossa staining, Field Emission-Scanning Electron Microscopy with Energy Dispersive X-ray analysis, $\mu$-Fourier Transform Infrared Spectroscopy, Cryo-Transmission Electron Microscopy coupled to Selected-Area Electron Diffraction and Electron Energy Loss Spectroscopy.

Results: Incipient Randall's plaque has been observed in $72.7 \%$ of kidneys. Carbonated apatite was as expected the main component of microcalcifications but amorphous calcium phosphate and whitlockite have been identified in $80 \%$ and $40 \%$ of the papillae, respectively. Incipient plaques stood in the deepest part of the papillae, around the loop of Henle tip but also around vasa recta (respectively $62.4 \%$ and $37.2 \%$ of microcalcifications) and rarely close to collecting ducts. At the nanoscale level, incipient calcifications were often made of several nanocrystals inside organic material looking like microvesicles.

Conclusions: Incipient Randall's plaque is frequent and appears at the extreme tip of renal papillae, around the hairpin structure of the loop of Henle but also around vasa recta. Nanoscale analyses suggest a local nucleation process promoting nanocrystal growth in a supersaturated milieu. In addition, plaques contain various calcium and magnesium phosphates and not only carbonated apatite. 


\section{Introduction}

Alexander Randall proposed in 1936 an original theory relative to "The origin and growth of renal calculi". ${ }^{1,2} \mathrm{He}$ was the first to identify at the tip of renal papillae interstitial calcium phosphate $(\mathrm{CaP})$ deposits whose growth resulted in urothelium rupture. He observed "Randall's plaques" in $17 \%$ of kidneys, and small calcium oxalate stones were found to be attached to plaque at the tip of papillae in 65 kidneys $(2.8 \%){ }^{2}$ Calcium oxalate stones expelled by patients had in some cases a depression due to papilla imprinting and $\mathrm{CaP}$ residues, thereby involving plaques as the origin of stones. During the same period, Rosenow observed plaques in $22 \%$ of 239 kidneys and Vermooten in $17.2 \%$ of Caucasian patient kidneys. ${ }^{3-5}$ Despite these seminal works, Randall's plaque generated a limited interest during the second part of the XXth century. ${ }^{6-8}$

A major contribution in the Randall's plaque dedicated research has been performed by Evan et al. in 2003. ${ }^{9}$ They performed kidney biopsies in renal stone formers and observed Randall's plaque at the tip of renal papillae, made of calcium-containing deposits surrounding loops of Henle. These deposits were identified as made of apatite. Electron microscopy evidenced that they were located in the basement membrane of loop of Henle but not in cell cytoplasm.

The mechanisms underlying the formation of apatite deposits remain elusive. Asplin et al. have hypothesized that $\mathrm{CaP}$ supersaturation in the thin limb of loop of Henle would promote CaP particle precipitation. ${ }^{10}$ Some tubular crystals attached to epithelial cells could undergo an endocytosis process and precipitate in basement membranes. ${ }^{11}$ Once initiated, the interstitial calcification process extends in the interstitium, forming plaques made of apatite at the contact of macromolecules such as osteopontin. ${ }^{12}$

To date, biopsies have been performed in renal stone formers affected by large plaques and plaque frequency and structure has been analyzed in autopsic series, therefore in old patients. Since Randall's plaque formation has been observed in non-stone formers by renoureteroscopy, we hypothesized that incipient Randall's plaque would not be an exception in kidneys. We analyzed the healthy papillae of kidneys removed for cancer (i) to assess the frequency of incipient Randall's plaque in living non-stone formers, (ii) to identify the crystalline phases responsible for plaque formation, and (iii) to characterize at the nanometer scale their initial formation site and their structure. We identified nascent Randall's plaques in a majority of papillae, made of various phosphate phases, around loops of Henle and vasa recta and not around collecting ducts. 


\section{Patients and Methods}

\section{Papilla collection}

We studied kidneys removed for cancer from 22 patients $(67.7 \pm 10.5$ years old) between 2012 and 2014. Papillae from healthy parts of the kidney, at distance from the tumor, have been collected and coded anonymously after extemporaneous examination. Patients gave a written consent relative to kidney removal and tissue conservation but not to clinical or biological data. Papillae have been therefore anonymously collected, according to French legislation and Helsinki declaration for Patient Safety. Half papillae have been embedded in paraffin after fixation by paraformaldehyde. The other half papilla has been frozen or fixed with glutaraldehyde to perform electron microscopy. No attached stone has been observed at the surface of the papilla and, a priori, there was no stone former among the patients.

\section{Von Kossa staining and immunohistochemical analyses}

Four- $\mu \mathrm{m}$ tissue sections have been performed and stained by Von Kossa procedure to reveal tissue calcifications. To assess the topography of incipient plaques, Von Kossa staining has been followed by classical immunostaining. Endothelial cells were immunostained with mouse anti-human CD34 antibody (1/800, clone QBend/10, Euro-Diagnostica, Sweden), revealing thereby capillaries of the papilla (vasa recta). Collecting ducts were immunostained with goat anti-human aquaporin 2 antibody (1/100, clone C-17, Santa Cruz biotechnology, USA). Samples were revealed with Single Stain MAX PO (mouse or goat) Histofine (Nichirei Biosciences, Japan).

\section{Field Emission-Scanning Electron Microscopy and Energy Dispersive X-ray (FE-SEM- EDX)}

Four- $\mu \mathrm{m}$ tissue sections were investigated with a Zeiss SUPRA55-VP Field Emission scanning Electron Microscopy (FE-SEM). Measurements were performed at low voltage $(1.4 \mathrm{KeV})$ and without the usual deposits of carbon at the surface of the sample. Energy Dispersive X-ray (EDX) experiments were performed, allowing calcium and phosphate distribution cartography. The FE-SEM operated at $12 \mathrm{keV}$ to perform calcium and phosphate cartography.

\section{$\mu F T I R$ spectroscopy}


Microcalcification phases were characterized using $\mu$ Fourier Transform InfraRed spectrometry. Four micrometer tissue sections were deposited on low emission microscope slides (MirrIR, Keveley Technologies, Tienta Sciences, Indianapolis). FT-IR hyperspectral images were recorded with a Spectrum spotlight 400 FT-IR imaging system (Perkin Elmer Life Sciences, France), with a spatial resolution of $6.25 \mu \mathrm{m}$ and a spectral resolution of $8 \mathrm{~cm}^{-}$ 1. The spectra were recorded in the $4000-700 \mathrm{~cm}^{-1}$ mid-InfraRed range. Each spectral image, covering a substantial part of the tissue, consisted of about 30,000 spectra.

\section{Transmission electron microscopy (TEM)}

Small pieces of papilla tip were fixed in $2.5 \%$ glutaraldehyde in $0.1 \mathrm{mmol} / \mathrm{L}$ cacodylate buffer $(\mathrm{pH} 7.4)$ at $4^{\circ} \mathrm{C}$. Fragments were then post-fixed in $1 \%$ osmium tetroxide, dehydrated using graded alcohol series, and embedded in epoxy resin. Semi-fine sections $(0.5 \mu \mathrm{m})$ were stained using toluidine blue. Ultrastructure sections $(80 \mathrm{~nm})$ were contrast-enhanced using uranyl acetate and lead citrate, and examined using a JEOL 1010 electron microscope (JEOL, Ltd., Tokyo, Japan) with a MegaView III camera (Olympus Soft Imaging Systems GmbH, Münster, Germany).

\section{Electron Energy Loss Spectroscopy (EELS) experiments}

EELS experiments were performed in a VGHB01 Scanning Transmission Electron Microscope (STEM) equipped with a cold field emission gun operated at $100 \mathrm{keV}$. EELS data were collected on a low noise / low temperature CCD camera (Princeton Instruments) optically coupled to a scintillator in the image plane of a Gatan 666 magnetic sector. The sample was cooled down to liquid-nitrogen temperature using a home-made cryo stage.

\section{CryoTEM experiments}

Experiments were performed on a JEOL 2010 TEM microscope equipped with a GATAN 626 cryo holder. The microscope was operated at $200 \mathrm{keV}$. Images and selected area diffraction patterns were collected using a Gatan Orius CCD camera.

\section{$\underline{\text { Results }}$}

\section{Incipient Randall's plaque is frequent in non-stone formers}

Among 22 kidneys removed for cancer, we analyzed 54 papillae in the healthy parts of the kidney (median of 2.5 papilla/patient). Von Kossa staining of kidney tissues identified two frequent types of renal calcifications, (i) tubular plugs, which were present in $43(79.6 \%)$ 
papillae, at various levels, and (ii) distal round-shaped structures or incipient Randall's plaque in $34(63 \%)$ papillae, at the tip of the papilla (Figures 1A-D). Overall, incipient Randall's plaques were present in 16 patients $(72.7 \%)$. Among the 22 patients, 14 had both plaques and tubular plugs, 2 had plaques only, 4 had intratubular plugs only and 2 had no evidence of kidney calcifications (Figure 1D). Among the 6 patients without plaques, 4 had tubular plugs whereas among the 16 patients with plaques, 14 had tubular plugs ( $p=0.29$, Fisher's exact test). When present, incipient plaques were detectable in all the collected papillae in 14 patients, $1 / 4$ papillae and 1/3 papillae in the 2 other patients respectively, suggesting that the development of Randall's plaques often involves several papillae at the same time in an individual. We did not evidence any massive plaque harbouring kidney stone. Interestingly, there was no spatial correlation between tubular plugs and incipient plaques (Figure 1C).

\section{Composition of incipient Randall's plaque}

To assess whether structures revealed by Von Kossa staining were actually crystalline deposits, we performed FE-SEM-EDX in five papillae. Spheroid deposits were either isolated or formed rings around tubular structures, and contained high amounts of calcium and phosphate (Figure 2A-E). To go further in the characterization of the crystalline phases, we performed $\mu$ Fourier Transform InfraRed ( $\mu$ FTIR) spectroscopy with an imaging system on 10 papillae removed from 10 kidneys. The analysis of the absorption spectrum and its second derivative revealed some features specific for the presence of different absorption bands of the apatite $\left[\mathrm{Ca}_{5}\left(\mathrm{PO}_{4}\right)_{3}(\mathrm{OH})\right]$, especially the $v_{3} \mathrm{P}-\mathrm{O}$ stretching vibration mode measured at 1035 $1045 \mathrm{~cm}^{-1}$. Of notice, carbonate ions were detected together with apatite by their $v_{3} \mathrm{C}-\mathrm{O}$ stretching vibration mode around $1420 \mathrm{~cm}^{-1}$ and the $v_{2} \mathrm{C}-\mathrm{O}$ bending mode at $875 \mathrm{~cm}^{-1}$. Carbonated apatite or carbapatite has been identified in 9 papillae (90\%) (Figures 3A, 3B and 3D).

In addition, the presence of amorphous calcium phosphate (ACP), revealed by the partial disappearance of the shoulder of the $v_{3} \mathrm{P}-\mathrm{O}$ absorption band of apatite, has been observed in 8 (80\%) papillae (Figures 3B and 3D). At last, the presence of whitlockite, a calcium and magnesium phosphate revealed by typical absorbance peaks at $1078-1080$ and $990 \mathrm{~cm}^{-1}$ has been observed in $4(40 \%)$ patients (Figures 3C-D). Tubular plugs contained mostly ACP and apatite but no whitlockite. 
The incipient Randall's plaque stood at the tip of renal papilla beneath the urothelium. Various levels of early plaques have been observed around lumens, forming most often continuous, circular structures, (Figure 1 A-D). To identify the structures surrounded by $\mathrm{CaP}$ deposits, immunohistochemistry analyses coupled to Von Kossa staining have been developed. The identification of collecting ducts has been performed with Aquaporin 2 specific antibodies (Figures 4A-B). Among 11 papillae, more than 1134 circular structures were surrounded by crystalline deposits according to Von Kossa staining but only $4(0.4 \%)$ of these lumens were collecting ducts identified by anti-aquaporin 2 antibodies (Table I).

The identification of capillaries/vasa recta has been performed by anti-CD34 (and anti-CD31, not shown) staining. Overall, among 11 papillae, 422 structures surrounded by crystalline deposits $(37 \%)$ were vasa recta (Table I). In a very large majority of capillaries, the calcium crystallites formed a circle around the lumen without any continuity with other interstitial calcifications (Figures 4C-D).

Immunochemistry studies were also performed using antibodies specific for antigens present in the descending or the ascending loop of Henle (HNK1, CLC-Ka, Aquaporin 1, Barttin, NKCC2) or common to vessels and tubules (urea transporters). Descending and ascending loops have been identified by these antibodies in the medulla but not at the tip of the papilla (not shown) and we had no specific marker of the hairpin structure of the loop of Henle. We considered therefore that papillary tubular structures which were not the easily recognized collecting ducts and not identified by anti-CD-34 antibodies would necessarily be deepest parts of the loops of Henle. Therefore, among 11 papillae, 708 structures surrounded by crystalline deposits (62\%) were identified as loops of Henle (Table 1).

\section{Micrometer and nanometer scale characterization of incipient plaques}

We first analyzed incipient plaques and early "carbapatite" deposits at the micrometer scale by using transmission electron microscopy. Electron dense deposits were observed around both capillaries and loops of Henle and predominated in the interstitium and at the external part of the basement membrane lamina densa. These microcalcifications were in many cases in close contact with collagen bundles (Figure 5A and 5B). Three types of structures have been observed: large, concentric and sometimes laminated structures but also small dense and homogeneous structures measuring 0.1-0.3 $\mu \mathrm{m}$ in diameter and less dense round shaped structures of various size looking like microvesicles (Figures 5A-E). Electron energy loss spectroscopy (EELS) analyses confirmed at the nanometer scale that dense deposits observed by electron microscopy were mainly made of $\mathrm{CaP}$ (figures 5D-F). Interestingly, many round 
shaped structures looked like microvesicles with an electron dense "membrane" measuring about 8-10 $\mathrm{nm}$. EELS analyses evidenced calcium phosphate nanoparticles aggregates in the center of the structure and surprisingly lack of calcium and phosphate but high amounts of nitrogen at the "membrane" (Figure 5D-F).

To go further into the characterization of the microcalcifications and determine their crystalline nature, Cryo-transmission electron microscopy (Cryo-TEM) coupled to SelectedArea Electron Diffraction (SAED) has been performed. We evidenced that "microvesicles" contained a few nanocrystals whose diffraction pattern supported the presence of crystalline apatite. We could not dissociate whitlockite and apatite diffraction patterns due to the low amount of material (Figure 6 A-C). Nanocrystals predominated in the center of microvesicles (Figure 6 B).

\section{Discussion}

In his seminal work, Alexander Randall evidenced papillary lesions in one or more of the renal papillae in 73 of 429 pairs of cadaveric kidneys $(17 \%) .{ }^{1,2}$ The development of renoureteroscopy and morphoconstitutional kidney stone analysis highlighted a potential increase in the incidence of Randall's plaque during the past decades. ${ }^{13}$ Low and Stoller reported in 1997 the presence of Randall's plaques in $74 \%$ of 57 patients with stone and $43 \%$ of 7 patients without stones. ${ }^{14}$ In France, Daudon and Traxer found Randall's plaques in $57 \%$ of 287 stone formers and $27 \%$ of 173 non-stone forming patients. ${ }^{15}$ Our results evidence that incipient plaque affects more than two thirds of the kidneys analyzed.

$\mathrm{CaP}$ supersaturation at the tip of renal papillae has been recognized for a long time. ${ }^{16} \mathrm{~A}$ medullary concentration gradient for calcium has been described, potentially promoting Randall's plaque growth. ${ }^{17,18}$ Kuo et al. have shown that hypercalciuria and low diuresis were associated to the surface by Randall's plaque in renal stone formers. ${ }^{19}$ Another important issue is the interstitial $\mathrm{pH}$ at the tip of the papilla. but only indirect data suggest that papillary $\mathrm{pH}$ may be relatively high in patients affected by Randall's plaque. ${ }^{20}$

Evan et al. evidenced that the initial site of Randall's plaque begins in basement membrane of thin loops of Henle and apatite spreads from there through the interstitium to beneath the urothelium but the origin of $\mathrm{CaP}$ microcalcifications has been discussed. ${ }^{9}$ It has been hypothesized that small tubular $\mathrm{CaP}$ crystals might be transported through transcellular or intercellular way to the interstitium. Another hypothesis would be an internalization and intracellular dissolution of tubular $\mathrm{CaP}$ deposits resulting in an increased basolateral excretion of calcium and phosphate ions. At last, it has been hypothesized that interstitial $\mathrm{CaP}$ 
supersaturation and high $\mathrm{pH}$ would promote in situ interstitial CaP precipitation. ${ }^{9,}{ }^{21-23}$ The observations we made support the latter hypothesis. Actually, we observed incipient plaques around loops of Henle but also around vasa recta at the tip of the papilla and argue for an initial formation of interstitial deposits rather than a migration of tubular crystals across epithelial cells. In addition, tubular plugs made of carbapatite and ACP were not in contact with incipient plaques. At last, the observation of interstitial CaP nanocrystals surrounded by "microvesicles" suggests a growth process arising locally rather than a migration of $\mathrm{CaP}$ crystals. According to the first detailed mathematical model of calcium transport along the nephron (in the rat kidney), the permeability of vasa recta to calcium would promote interstitial CaP supersaturation in their vicinity. ${ }^{24}$

The topography of incipient plaques and especially the contribution of collecting ducts to plaque formation is also a matter of debate. On the one hand, Stoller et al. analyzed cadaveric kidneys and identified small spherical calcifications condensed into rings around collecting ducts and their tributaries, by using CAM 5.2 (anti-keratin) antibody, and to a lesser extent around vascular structures identified by anti CD-34 antibodies. ${ }^{25}$ On the other hand, Evan et al. described the presence of plaques around loops of Henle but observed no evidence of plaque around collecting ducts. ${ }^{9}$ Our quantitative analyses identified almost no calcifications around collecting ducts (revealed by anti-aquaporin 2 antibodies), but frequent round-shaped calcifications around vasa recta and loops of Henle. Of notice, we analyzed small incipient calcifications from "relatively" young nephrectomized patients whereas Stoller et al. analysed kidneys from deceased and therefore probably older patients with radiographically evident plaques, that may spread toward collecting ducts.

An interesting finding is the diversity of microcalcifications at the nanoscale level. Khan et al. have previously reported the presence of calcified vesicles in plaques.$^{26}$ It has been evidenced during the past years that Randall's plaque microcalcifications contain high amounts of calcium-binding proteins, the leader being osteopontin. ${ }^{27}$ The observation of concentric structures highlights the close relationships between proteins and mineral phases in these structures. $^{28}$ The hypothesis that "microvesicles" would be the first step toward plaque formation is supported by the observation of nanocrystals inside round-shaped structures with a "membrane-like" structure with a thickness of 8-10 nm. Surprisingly, these "membranes" were electron dense but contained low amounts of calcium and phosphorus, and high amounts of nitrogen. These observations deserve further studies to assess whether these round shaped structures are accumulation of proteins (osteopontin) or plasma membranes preceding the formation of the classical concentric laminated structures forming Randall's plaque. 
The analysis of incipient plaques evidences that apatite is not the sole mineral involved in plaque formation. The presence of ACP supports the hypothesis that microcalcifications rise in situ. Interestingly, magnesium-containing whitlockite has been identified in several kidneys. Whitlockite has been identified recently in vascular calcifications in uremic patients. ${ }^{29}$ In a rat model of uremic calcifications, the presence of whitlockite has been exclusively found in vitamin D-treated animals, probably due to vitamin D-stimulated gastrointestinal absorption of magnesium. ${ }^{30}$ Interestingly, sensitivity to vitamin D may promote Randall's plaque, ${ }^{13}$ Nevertheless, we did not collect biological or clinical data from patients, due to study design. This is one of the main limitations of the study. For instance we have no data about their vitamin D status or sensitivity to calcitriol, and we may only presume that patients were not stone formers. Secondly, we presumed that nascent plaques are the first step toward potentially larger plaques from which stones originate but we have no idea about the natural history of plaque formation. In the third place, we have no specific marker of the hairpin structure of the loop of Henle. However, it seems likely that tubular structures present in deepest part of the papilla, which are not collecting ducts and vessels, belong to the deepest part of the nephron.

In conclusion, the analysis of incipient plaques at the nanoscale level provides original informations. Firstly, incipient Randall's plaque is an extremely frequent process and appears at the tip of the papilla. In addition, Randall's plaque elementary structure is not uniform and we observed nanocrystals surrounded by organic structures, possibly inside vesicles, but also smaller dense deposits whose organization around vessels at the microscopic level suggests that biomineralization is promoted by $\mathrm{CaP}$ local interstitial supersaturation, probably driven by vasa recta. At last, plaques are not made only of carbonated apatite and the presence of whitlockite deserves further studies dedicated to the role of magnesium and vitamin D in plaque formation.

\section{Fundings}

This work has been supported by the Agence Nationale de la Recherche (ANR-13-JSV10010-01, ANR-12-BS08-0022), the Société de Néphrologie (Genzyme grant), the Académie 
Nationale de Médecine (Nestlé-Waters award), Convergence-UPMC CVG1205 and CORDDIM-2013-COD130042.

Cécile Verrier has been supported by the Association Française d'Urologie (AFU). 


\section{References}

1. Randall A: An hypothesis for the origin of renal calculus. N Engl J Med 1936; 214: 234.

2. Randall A: The origin and growth of renal calculi. Ann Surg 1937; 105: 1009.

3. Rosenow EC Jr: Renal calculi: study of papillary calcification. J Urol 1940; 44: 19.

4. Vermooten V: The incidence and significance of the deposition of calcium plaques in the renal papilla as observed in the Caucasian and Bantu population in South Africa. J Urol 1941; 46: 193.

5. Vermooten V: Origin and development in renal papilla of Randall's calcium plaques. J Urol 1942; 48: 27.

6. Anderson L, Mc Donald JR: The origin, frequency, and significance of microscopic calculi in the kidney. Surg Gynecol Obstet 1946; 82: 275.

7. Cifuentes-Delatte L, Minon-Cifuentes J, Medina JA: New studies on papillary calculi. J Urol 1987; 137: 1024.

8. Cifuentes Delatte L, Minon-Cifuentes JL, Medina JA: Papillary stones: calcified renal tubules in Randall's plaques. J Urol 1985; 133: 490.

9. Evan AP, Lingeman JE, Coe FL, et al: Randall's plaque of patients with nephrolithiasis begins in basement membranes of thin loop of Henle. J Clin Invest 2003; 111: 607.

10. Asplin JR, Mandel NS, Coe FL: Evidence of calcium phosphate supersaturation in the loop of Henle. Am J Physiol 1996; 270: F604.

11. Lieske JC, Norris R, Swift H, et al: Adhesion, internalization and metabolism of calcium oxalate monohydrate crystals by renal epithelial cells. Kidney Int 1997; 52: 1291.

12. Evan AP, Coe FL, Lingeman JE, et al: Mechanism of formation of human calcium oxalate renal stones on Randall's plaque. Anat Rec (Hoboken) 2007; 290: 1315.

13. Letavernier E, Vandermeersch S, Traxer O, et al: Medicine (Baltimore). 2015; 94: e566.

14. Low RK, Stoller ML: Endoscopic mapping of renal papillae for Randall's plaques in patients with urinary stone disease. J Urol 1997; 158: 2062.

15. Daudon M, Traxer O, Williams JC, et al: Randall's plaques. In : Urinary tract stone disease edited by Rao PN, Preminger GM, Kavanagh JP, Springer, London; 2011:103. 
16. Hautmann R, Lehmann A, Komor S: Intrarenal distribution of oxalic acid, calcium, sodium and potassium in man. Eur J Clin Invest 1980; 10: 173.

17. Martin M, Hadj Aissa A, et al: Intrarenal distribution of calcium and magnesium in the dog. Effect of an osmotic diuresis. J Physiol (Paris) 1975; 70: 159.

18. Worcester EM, Coe FL: New insights into the pathogenesis of idiopathic hypercalciuria. Semin Nephrol 2008; 28: 120.

19. Kuo RL, Lingeman JE, Evan AP, et al: Urine calcium and volume predict coverage of renal papilla by Randall's plaque. Kidney Int 2003; 64: 2150.

20. Tiselius HG: Solution chemistry of supersaturation. In: Kidney Stones: Medical and Surgical Management. Edited by Coe FL, Favus MJ, Pak CYC, Parks JH, Preminger GM, Lippincott Raven Publishers, Philadelphia; 1996: 33.

21. Parks JH, Worcester EM, Coe FL, et al: Clinical implications of abundant calcium phosphate in routinely analyzed kidney stones. Kidney Int 2004; 66: 777.

22. Tiselius HG: The role of calcium phosphate in the development of Randall's plaques. Urolithiasis 2013; 41: 369 .

23. Khan SR: Role of renal epithelial cells in the initiation of calcium oxalate stones. Nephron Exp Nephrol 2004; 98: e55.

24. Tournus M, Seguin N, Perthame B, et al: A model of calcium transport along the rat nephron. Am J Physiol Renal Nephrol 2013; 305: F979.

25. Stoller ML, Low RK, Shami GS et al: High resolution radiography of cadaveric kidneys: unraveling the mystery of Randall's plaque formation. J Urol 1996; 156: 1263.

26. Khan SR, Rodriguez DE, Gower LB, et al: Association of Randall plaque with collagen fibers and membrane vesicles. J Urol 2012; 187: 1094.

27. Evan AP, Coe FL, Rittling SR, et al: Apatite plaque particles in inner medulla of kidneys of calcium oxalate stone formers: osteopontin localization. Kidney Int 2005; 68: 145.

28. Amos FF, Dai L, Kumar R, et al: Mechanism of formation of concentrically laminated spherules: implication to Randall's plaque and stone formation. Urol Res 2009; 37: 11.

29. Schlieper G, Aretz A, Veberckmoes SC, et al: Ultrastructural analysis of vascular calcifications in uremia. J Am Soc Nephrol 2010; 21: 689. 
30. Verberckmoes SC, Persy V, Behets GJ, et al: Uremia-related vascular calcification: more than apatite deposition. Kidney Int 2007; 71: 298. 


\section{Figure and Table Legends}

Figure 1: Randall's plaque and tubular plugs. Incipient plaques were stained with the Von Kossa technique (Figures 1A-B, magnification x100-mosaic and x400). Plaques were circular and differed from intratubular plugs (respectively white and black arrows, Figure 1C, magnification x100). Nascent plaques and tubular plugs were present in a majority of the 54 papillae from 22 kidneys (Figure 1D).

Figure 2: FE-SEM-EDX analysis of incipient plaques. Papilla areas affected by incipient plaques have been selected (Figure 2A, x400). FE-SEM performed on papilla slices revealed round-shaped and circular structures surrounding tubules (Figure 2B). EDX analysis revealed high proportions of calcium (Figures 2C and 2D) and phosphate (Figures 2C and 2E) which are co-localized (Figure 2F).

Figure 3: $\mu$ FTIR analysis and imaging of plaques. $\mu$ FTIR spectra revealed the presence of apatite mixed with amorphous calcium phosphate-ACP (Figures 3A-B) but also the presence of mixed apatite and whitlockite microcalcifications (Figures 3C and 3D).

Figure 4: Immunohistochemistry coupled to Von Kossa staining. Incipient plaques were almost never present around collecting ducts revealed by anti-aquaporin 2 antibodies (Figures 4A-B, magnification x100 and x400). By contrast, Incipient round shaped plaques were frequently present around vasa recta revealed by anti-CD-34 antibodies (Figures 4 C-D, magnification x100 and x400).

Figure 5: TEM and EELS analysis of incipient plaques. TEM revealed the presence of small dense deposits close to endothelial cells in contact with collagen bundles (Figures 5A-B, dark arrows) and "microvesicles" of various size (Figures 5A-B, white arrows). Largest concentric and laminated microcalcifications have also been observed (Figure 5A up right, same scale). High angle Annular Dark Field (HADF) images and EELS analysis revealed the presence of nanocalcifications made of $\mathrm{CaP}$ aggregated inside vesicles (Figures 5C-F) Figure 5D highlights a "microvesicle" containing nanocalcifications (top left, zoom: top right). Bottom left: EELS Ca cartography of the nanocalcifications. Bottom right: the false colour image is the superposition of EELS Ca cartography and HADF image. Figure 5E evidences the co-existence of microvesicles containing $\mathrm{CaP}$ crystals (NanoCal1) and smaller dense microcalcifications made of $\mathrm{CaP}$ with homogeneous structure (NanoCal2). Intermediate-sized structures containing also small nanocrystals have been identified 
(Nanocal3). Figure 5F: High-power magnification of NanoCal 1 (top left) and EELS cartography revealing that "membranes" were nitrogen-rich and contained low amounts of $\mathrm{CaP}(\mathrm{Ca}=$ calcium cartography, $\mathrm{P}=\mathrm{Phosphorus}$ cartography, $\mathrm{N}=$ Nitrogen cartography).

Figure 6: TEM at LN2 temperature and SAED analysis of incipient plaques. Bright field images showed microcalcifications (Figure 6A), whereas dark field image obtained using the most intense diffraction spots of the diffraction pattern confirmed that the nanoparticles in the center of "microvesicles" are crystalline (Figure 6B). Selected area diffraction pattern corresponding to microcalcifications have been studied (Figure 6C). The shortest distance corresponds to $\mathrm{Ca} .0 .53 \mathrm{~nm}$ and might be associated to either the (101) reflexion of apatite or the (110) reflexion of whitlockite. Both zone axis [-117] and [-23-2] of apatite and whitlockite matches the experimental diffraction pattern within several percents of accuracy, therefore preventing discrimination between both oxide phases (Figure 6C).

Table I: Number (N) and percentage (\%) of loops of Henle vasa recta and collecting ducts affected by incipient plaques revealed with Von Kossa staining $(\mathrm{VK}+)$ among 11 papillae. 


\begin{tabular}{|c|c|c|c|c|}
\hline & Papilla (N) & N & VK+(\%) & $\begin{array}{r}\text { Percentage of all VK+ } \\
\text { structures }\end{array}$ \\
\hline Loops of Henle & 11 & 2075 & $708(34.1)$ & $\mathbf{6 2 . 4}$ \\
\hline Capillaries & 11 & 3290 & $422(12.8)$ & $\mathbf{3 7 . 2}$ \\
\hline Collecting ducts & 11 & 352 & $4(1.1)$ & $\mathbf{0 . 3}$ \\
\hline
\end{tabular}




\section{A}

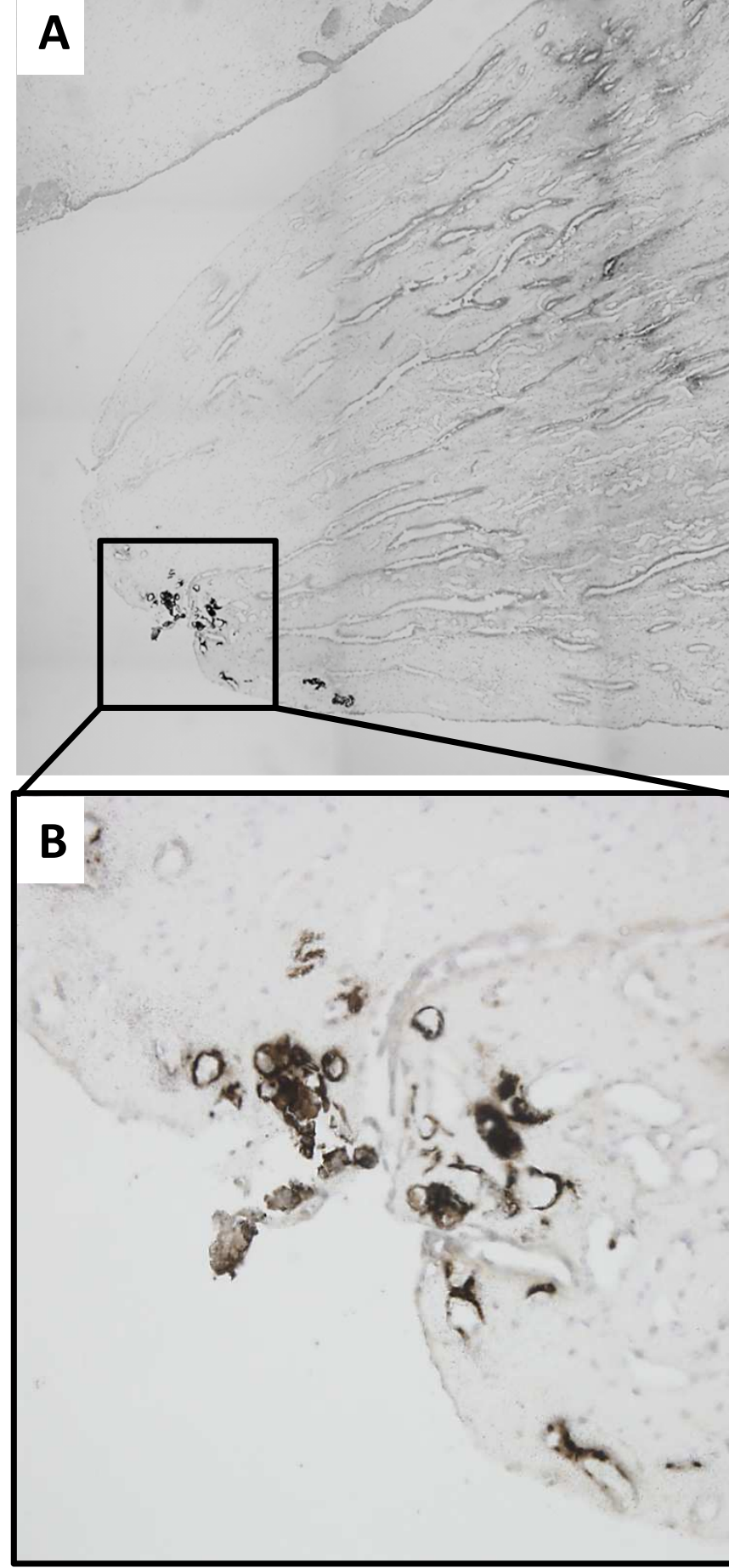

C
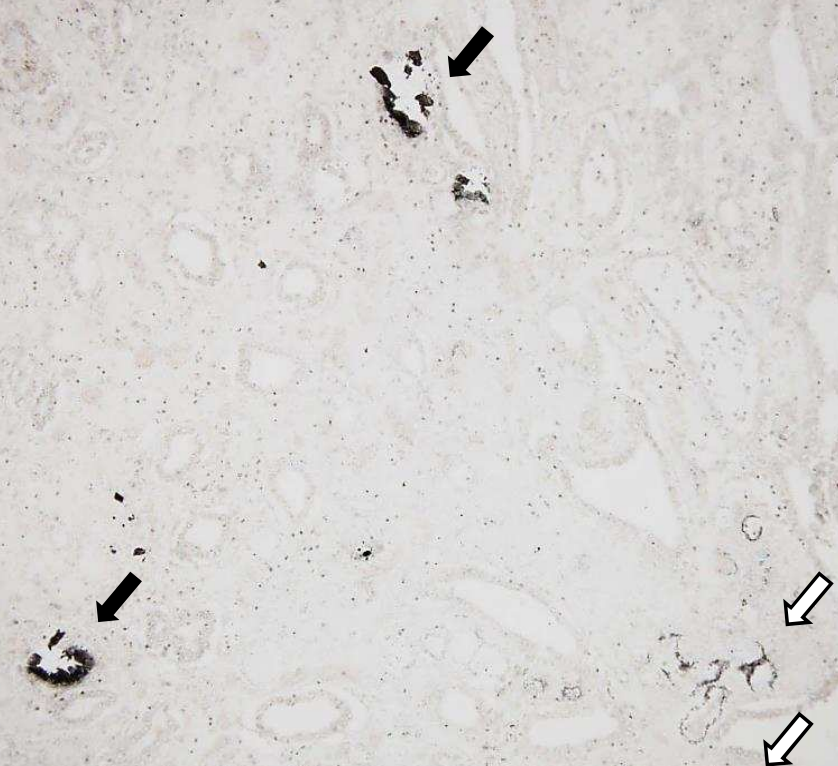

$\rightarrow$

D

\begin{tabular}{|c|c|c|c|c|c|}
\hline $\begin{array}{c}\text { Sam } \\
\text { ples } \\
\text { (N) }\end{array}$ & $\begin{array}{c}\text { Nascent } \\
\text { plaques only } \\
(\%)\end{array}$ & $\begin{array}{c}\text { Nascent plaques } \\
\text { + Tubular plugs } \\
(\%)\end{array}$ & $\begin{array}{c}\text { Tubular plugs } \\
\text { only } \\
(\%)\end{array}$ & $\begin{array}{c}\text { No } \\
\text { calcifications } \\
(\%)\end{array}$ \\
\hline Papillae & 54 & $7(13)$ & $27(50)$ & $16(30)$ & $4(7)$
\end{tabular}

Patients

22

2(9)

14(64)

$4(18)$

2 (9) 

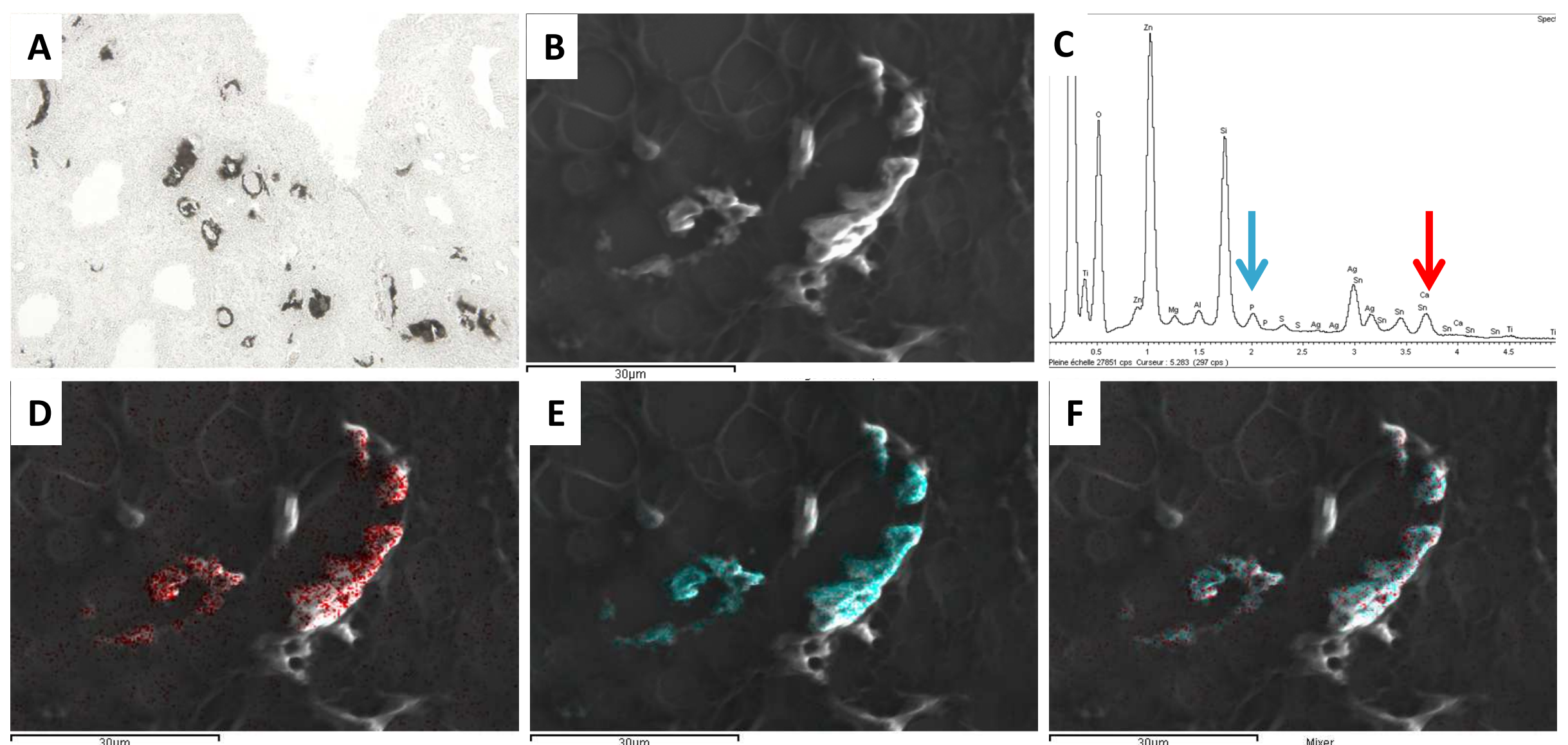

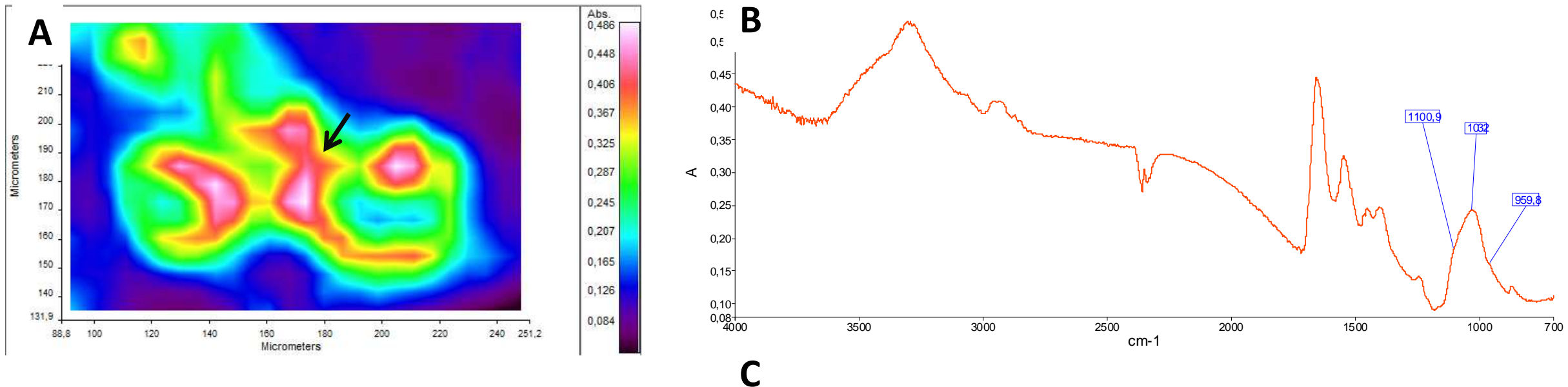

\section{D}

\begin{tabular}{|c|c|c|c|}
\hline & $\begin{array}{c}\text { Apatite } \\
(\%)\end{array}$ & $\begin{array}{c}\text { ACP } \\
(\%)\end{array}$ & $\begin{array}{c}\text { Whitlockite } \\
(\%)\end{array}$ \\
\hline $\begin{array}{c}\text { Papilla } \\
(\mathrm{N}=10)\end{array}$ & $\mathbf{9 ( 9 0 )}$ & $\mathbf{8 ( 8 0 )}$ & $\mathbf{4 ( 4 0 )}$ \\
\hline
\end{tabular}

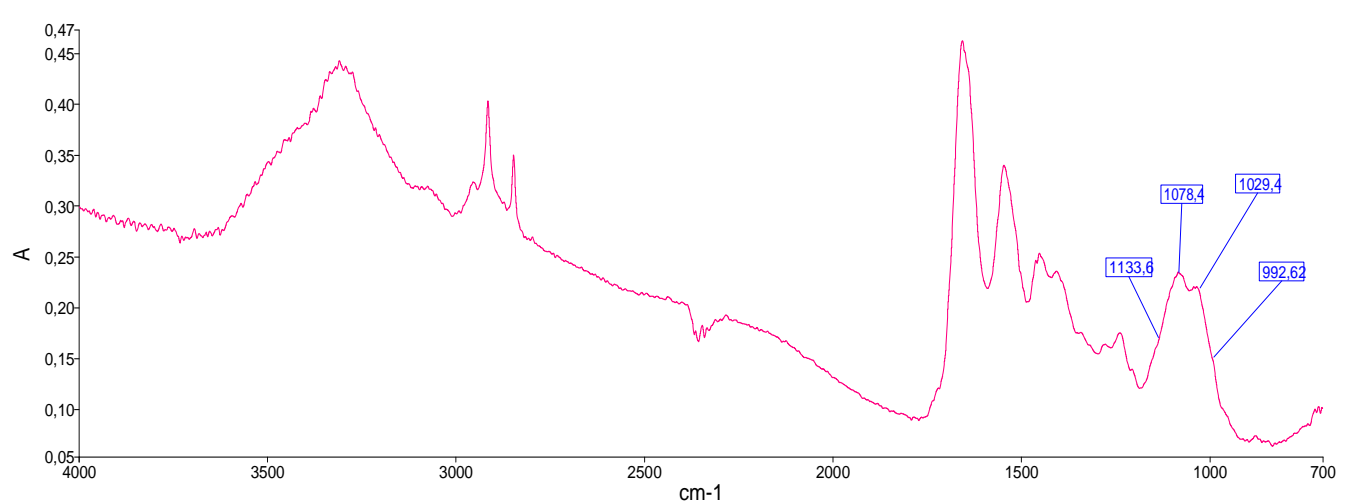



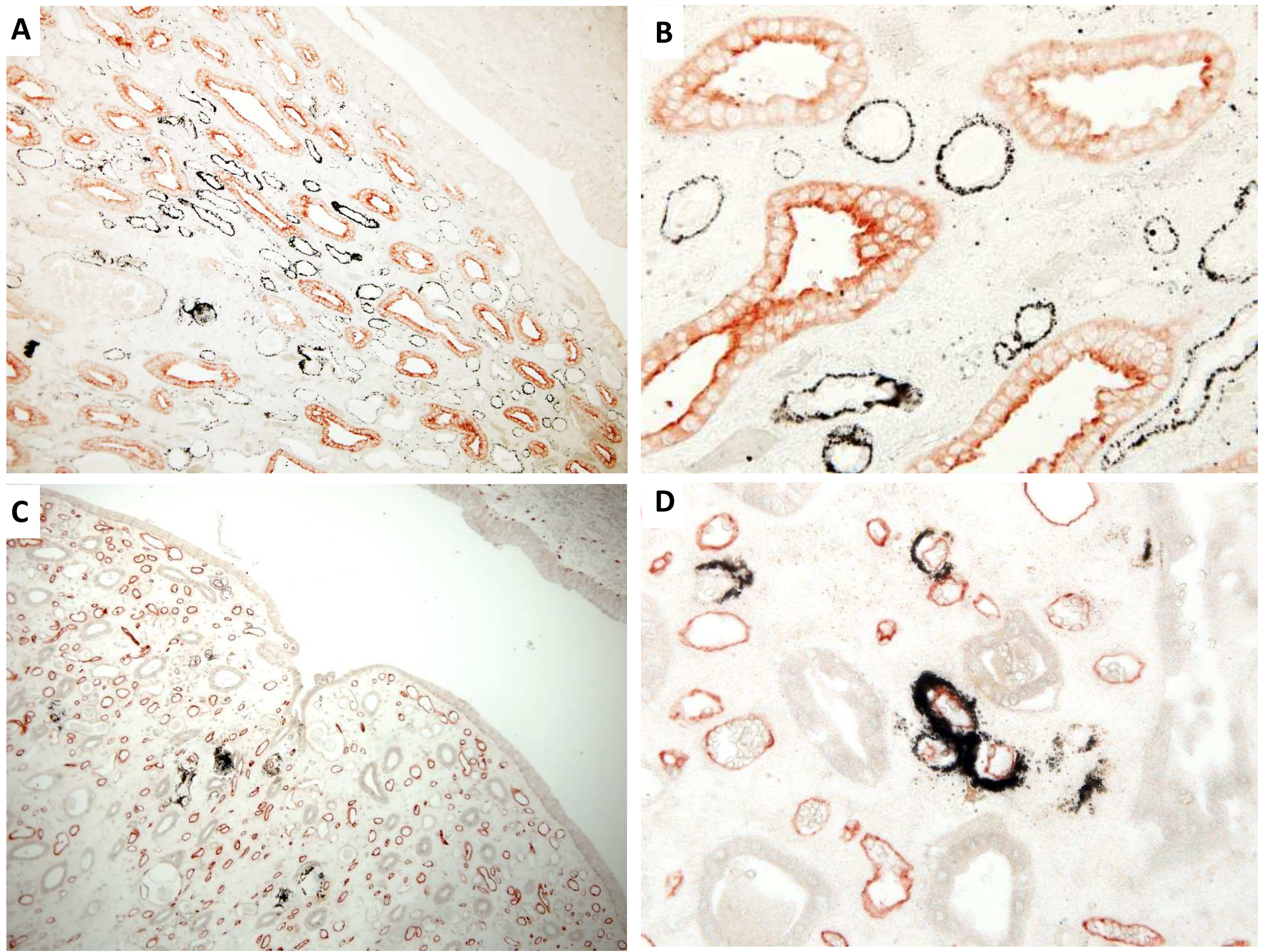

D

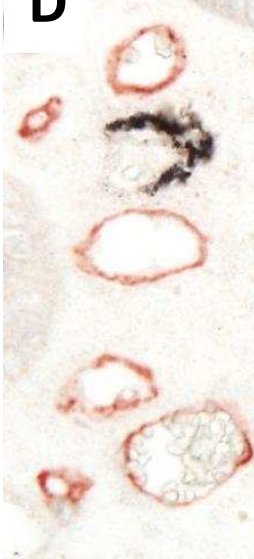

7
Q.

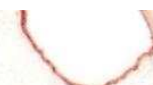

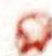

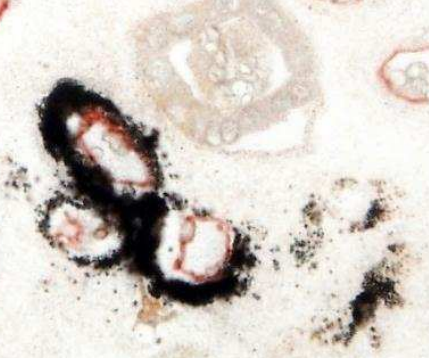

(3)

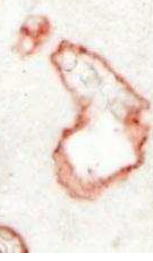


A

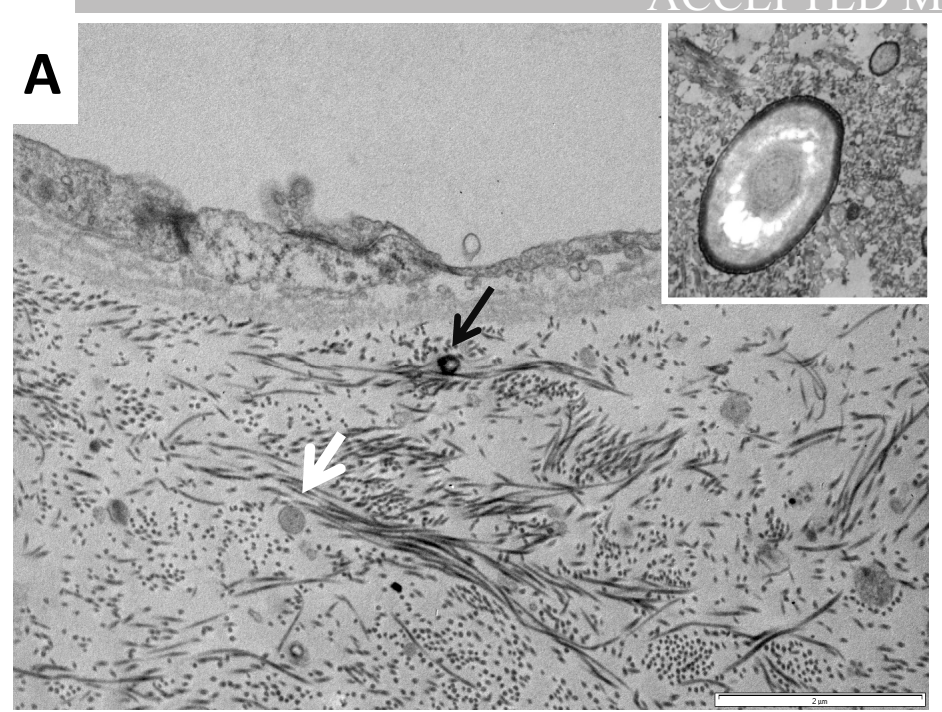

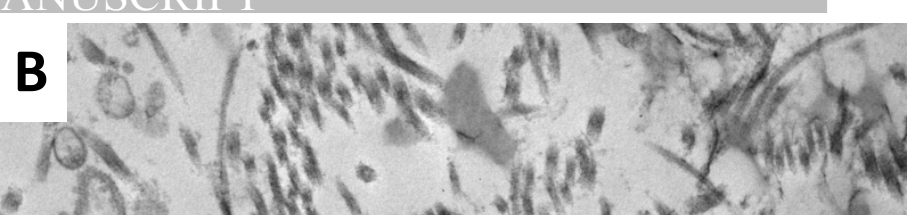
(2) ifla

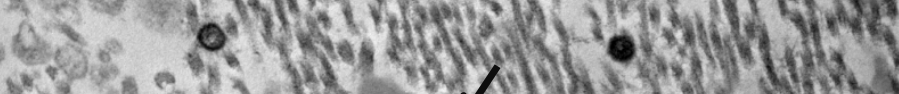

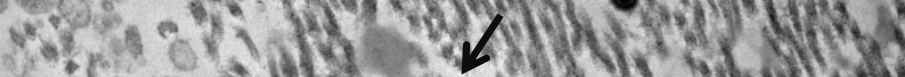

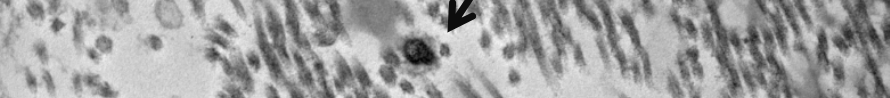

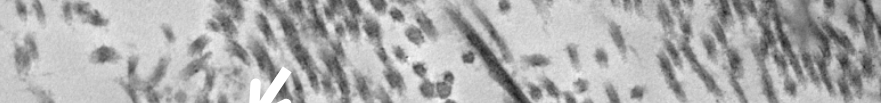

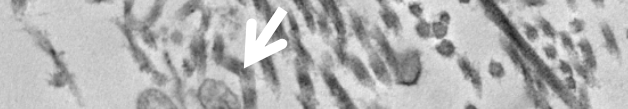
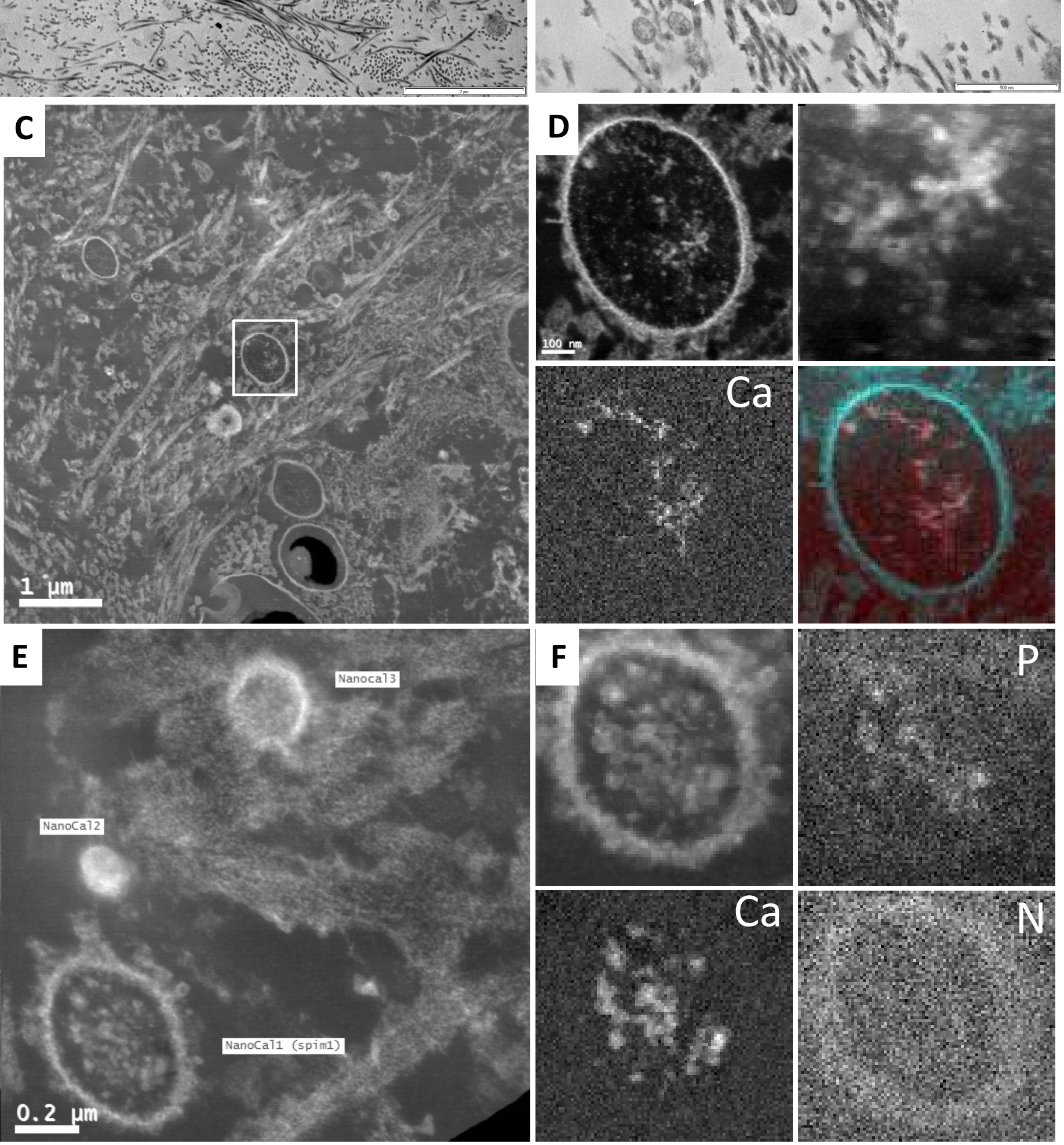

P.

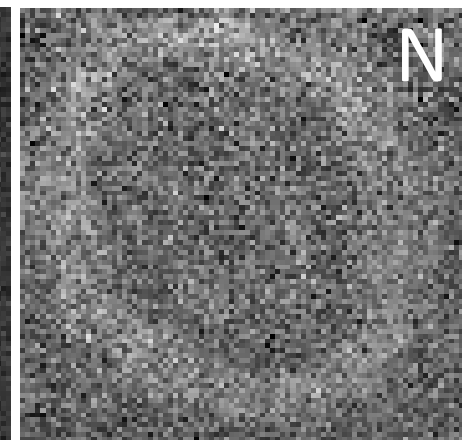




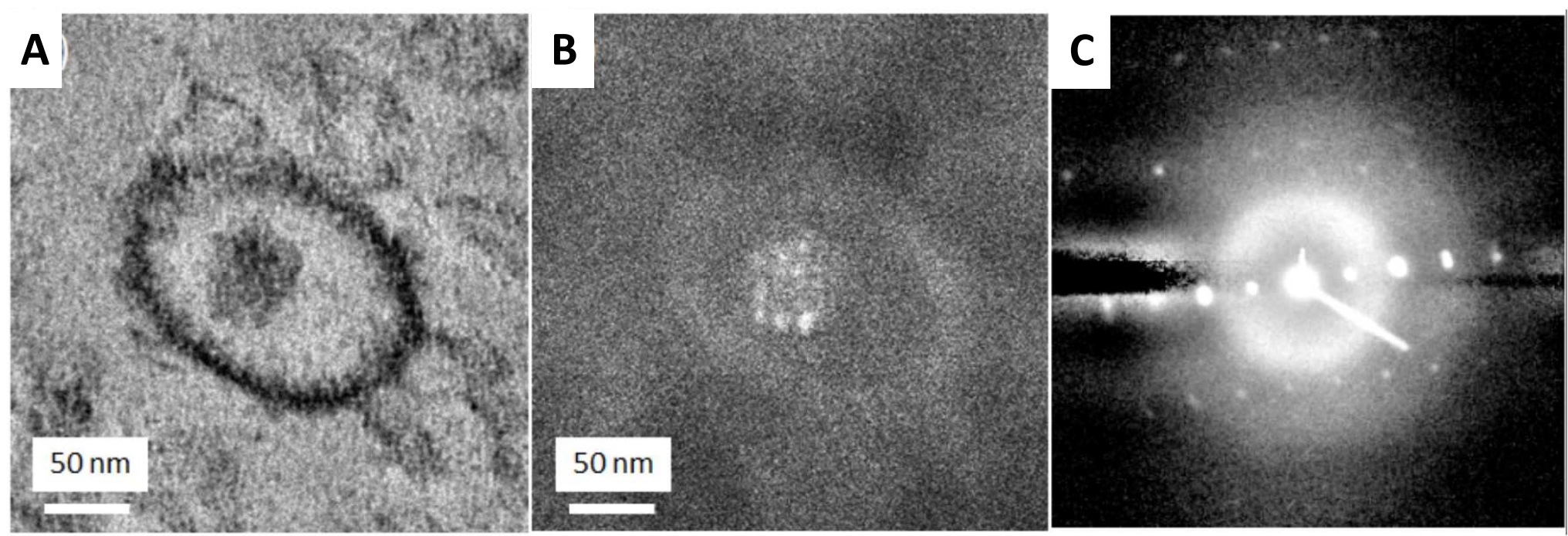


CaP : Calcium Phosphate

FE-SEM: Field Emission-Scanning Electron Microscopy

EDX: Energy Dispersive X-ray

FTIR: $\mu$ Fourier Transform InfraRed

EELS: Electron Energy Loss Spectroscopy 